\title{
PERSPECTIVA DE ENFERMERAS NEONATÓLOGAS SOBRE EL ACOMPAÑAMIENTO ESPIRITUAL AL FAMILIAR ANTE LA MUERTE DE UN PACIENTE NEONATO
}

\author{
PERSPECTIVE OF NEONATAL NURSES ON SPIRITUAL \\ ACCOMPANIMENT OF FAMILY MEMBERS IN THE FACE OF THE \\ DEATH OF A NEONATAL PATIENT
}

\section{PERSPECTIVA DE ENFERMEIRAS DE NEONATOLOGIA SOBRE O ACOMPANHAMENTO ESPIRITUAL AO FAMILIAR NA MORTE DE UM PACIENTE NEONATO}

\author{
Luz Milagros Saucedo Soberon* \\ Rosa Jeuna Díaz ManchaY** \\ Lisseth Dolores Rodríguez Cruz*** \\ Adela Rosanna Núñez Odar **** \\ Sonia Tejada Muñoz *****
}

\begin{abstract}
RESUMEN
Objetivo: Describir la perspectiva de enfermeras neonatólogas sobre el acompañamiento espiritual al familiar ante la muerte de un paciente neonato. Material y Método: Estudio cualitativo, descriptivo en una unidad de cuidados intensivos de un hospital público de la ciudad de Chiclayo, Perú. La muestra fue no probabilística y el muestreo por conveniencia. La saturación de los datos se alcanzó con 12 enfermeras. La recolección de datos se realizó entre agosto y septiembre 2019 mediante entrevista semiestructurada, previo consentimiento informado. El análisis de contenido fue mediante tres fases: Preanálisis, Codificación y Categorización. Se respetaron principios éticos de la Declaración de Helsinki y se tuvo en cuenta los criterios de rigor científico: Credibilidad, Confirmabilidad y Transferibilidad. Resultados: Se identificaron dos categorías: a) Trato humano, empatía y prácticas religiosas previas al deceso, b) Compasión y consuelo durante el fallecimiento. Conclusión: Se demuestra que la muerte de un paciente neonato en la unidad de cuidados intensivos es un suceso funesto e
\end{abstract}

*Licenciada en Enfermería. Escuela de Enfermería, Universidad Católica Santo Toribio de Mogrovejo, Chiclayo, Perú. ORCID: https://orcid.org/0000-0002-6846-4253. Email: milagros-0801@hotmail.com

**Doctora en Ciencias de Enfermería. Escuela de Enfermería de la Universidad Católica Santo Toribio de Mogrovejo, Chiclayo, Perú. ORCID: https://orcid.org/0000-0002-2333-7963. Email: rdiaz@usat.edu.pe.Autora de correspondencia.

***Maestra en Bioética. Escuela de Enfermería de la Universidad Católica Santo Toribio de Mogrovejo, Chiclayo, Perú. ORCID: https://orcid.org/0000-0003-1742-9498. Email: 1rodriguez@usat.edu.pe

****Maestra en Ciencias de Enfermería. Escuela de Enfermería de la Universidad Católica Santo Toribio de Mogrovejo, Chiclayo, Perú. ORCID: https://orcid.org/0000-0003-4334-6573. Email: anunez@usat.edu.pe

*****Doctora en Ciencias de Enfermería. Escuela de Enfermería de la Universidad Nacional Toribio Rodríguez de Mendoza, Chachapoyas, Perú. ORCID: https://orcid.org/0000-0002-1181-8540. Email: sonia.tejada@untrm.edu.pe 
inexplicable para padres/madres. El profesional de enfermería ofrece un acompañamiento espiritual basado en el trato humano, empatía, y permisión de prácticas religiosas como la oración y el bautismo; previo al suceso y al momento del fallecimiento se muestran compasivos y brindan consuelo a través de la esperanza en un ser superior, un abrazo y el permitir que la madre permanezca unos momentos con su bebé, antes de realizar la atención post mortem y los trámites administrativos.

Palabras clave: Espiritualidad; Enfermería neonatal; Familia; Muerte.

\begin{abstract}
Objective: To describe the perspective of neonatal nurses on the spiritual accompaniment of family members in the face of the death of a neonatal patient. Material and Method: Qualitative, descriptive study carried out in the intensive care unit of a public hospital in the city of Chiclayo, Peru. The sample was non-probabilistic and determined by using convenience sampling. Data saturation was reached with 12 nurses. Data collection was carried out between August and September 2019, using semi-structured interviews and prior informed consent. Content analysis was conducted in three phases: Pre-analysis, coding and categorization. The ethical principles of the Helsinki Declaration and the criteria of scientific rigor were considered: credibility, confirmability and transferability. Results: Two categories were identified: (a) humane treatment, empathy and religious practices before death, (b) compassion and comfort during death. Conclusion: The death of a neonatal patient in the intensive care unit is shown to be a fatal and incomprehensible event for parents. The nursing professional offers spiritual accompaniment, based on humane treatment, empathy and permission to perform religious practices such as prayer and baptism. Prior to the event and at the time of death, they show compassion and provide comfort through hope in a higher being, give a hug and allow the mother to stay a few moments with her baby, before performing postmortem care and administrative procedures.
\end{abstract}

Key words: Spirituality; Neonatal Nursing; Family; Death.

\title{
RESUMO
}

Objetivo: Descrever a perspectiva de enfermeiras de neonatologia sobre o acompanhamento espiritual do familiar diante da morte do paciente neonato. Material e Método: Estudo qualitativo descritivo em una unidade de terapia intensiva de um hospital público da cidade de Chiclayo, Peru. A amostra foi náo probabilística e por conveniência. A saturaçáo dos dados foi alcançada com 12 enfermeiras. Os dados foram coletados entre agosto e setembro de 2019 por meio de entrevista semiestruturada, com consentimento informado prévio. A análise de conteúdo ocorreu em três fases: Pré-análise, Codificação e Categorização. Foram respeitados os princípios éticos da Declaração de Helsinki e consideraram-se os critérios de rigor científico: Credibilidade, Confirmabilidade e Transferibilidade. Resultados: Duas categorias foram identificadas: a) Trato humano, empatia e práticas religiosas antes da morte, b) Compaixão e conforto durante a morte. Conclusão: Demonstra-se que a morte de um paciente neonato na unidade de terapia intensiva é um evento catastrófico e inexplicável para pais/ máes. $\mathrm{O}$ profissional de enfermagem oferece um acompanhamento espiritual baseado no trato humanizado, na empatia, e na permissão de práticas religiosas como a oraçáo e o batismo; antes do evento e no momento da morte, são compassivos e proporcionam conforto por meio da esperança em um ser superior, um abraço e permitir que a mãe permaneça alguns momentos com seu bebê, antes de realizar os cuidados pós-morte e os procedimentos administrativos.

Palavras-chave: Espiritualidade; Enfermagem neonatal; Família; Morte.

Fecha de recepción: 10/06/2020

Fecha de aceptación: 30/09/2020 


\section{INTRODUCCIÓN}

La Organización Mundial de la Salud ${ }^{(1)}$ reportó en el 2018 que el 47\% de las muertes de niños menores de 5 años son neonatos. En el Perú, en el $2017^{(2)}$, la mortalidad perinatal (muertes fetales y neonatales) fue de 14 defunciones por mil embarazos. A pesar de los avances tecnológicos y la especialización del profesional de salud, a veces es inevitable la muerte del neonato, así como la tristeza de los padres, sentimiento que es una respuesta natural frente a una pérdida y puede durar semanas, meses e incluso años ${ }^{(3)}$. Cuando los padres reciben la inesperada noticia de que su hijo ha muerto o está muriendo, generalmente experimentan un dolor profundo, miedo e incertidumbre ${ }^{(4)}$. Debido a ello, presentan un aumento significativo de problemas psíquicos y emocionales como la depresión, sensación de fracaso, ansiedad, ira, idea suicida y culpa ${ }^{(5)}$.

De acuerdo con especialistas, la fuerza y esperanza para afrontar y superar el dolor por la pérdida de un hijo derivan, justamente, del ámbito espiritual que les da el sentido de la vida; en esos momentos el aspecto religioso permite la conexión con el ser superior y trascendental, que facilita ese soporte espiritual ${ }^{(6)}$. El duelo es un suceso que conlleva a una búsqueda y un encuentro espiritual, siendo importante considerar que después de la muerte los familiares pueden permanecer un momento con el neonato y realizar ciertos rituales, como hacer arreglos del cuerpo o rendirle tributo ${ }^{(7)}$.

La cultura para expresar el dolor y para buscar consuelo en algunas prácticas religiosas es un aspecto importante que la enfermera de la Unidad de Cuidados Intensivos Neonatales (UCIN) debe conocer para brindar acompañamiento espiritual a los padres/madres en el momento de la muerte de su hijo. La principal necesidad espiritual descrita por los padres es mantener una conexión con su hijo en el momento de la muerte. Por ello, consideran importante la oración, el bautizo y la lectura de escrituras sagradas ${ }^{(8)}$. Entonces, el duelo es un proceso importante de cuidado, pues al comprender la continuación de la vida como una experiencia integral, la enfermera adquiere la capacidad de realizar una intervención trascendente y de valor para el ser humano que vive la situación, o para la familia que acompaña el proceso de enfermedad y muerte ${ }^{(9)}$.

La muerte de un neonato siempre es una situación difícil para las enfermeras, ya que forman un fuerte vínculo con el bebé y la familia; asimismo, reconocen que es más complicado aceptar la muerte de un recién nacido que de un adulto o un anciano ${ }^{(10)}$. Algunos estudios señalan que las enfermeras luchan por encontrar palabras para dialogar sobre el aspecto espiritual con una actitud de respeto, escucha y aliento, para acompañar a la familia a encontrar paz y fortaleza en sus vidas y aliviar, en alguna medida, la situación dolorosa ${ }^{(5,11)}$. Específicamente, esta temática es difícil de abarcar en los hospitales públicos de Chiclayo-Perú, sobre todo por la escasa dotación de personal y por los procedimientos tan complejos que se realizan día a día en las UCIN. A pesar de ello, cuando están frente a la inminente muerte de un paciente neonato, las enfermeras apoyan espiritualmente a los familiares, en especial a la madre.

Este estudio se justifica porque los cuidados espirituales tienen un gran valor en el ámbito de la salud y de la enfermería, especialmente ante situaciones dolorosas como la muerte de un hijo. Por esta razón, brindar un cuidado espiritual va más allá de un alivio físico o psicológico, en otras palabras, alivia internamente las heridas del sufrimiento, favoreciendo así el surgimiento de esperanza y tranquilidad, estados que ayudan a los padres/madres a encontrar sentido a la enfermedad o la muerte de su hijo. El aporte significativo de este estudio es que, ante situaciones dolorosas como la muerte de un hijo, el acompañamiento espiritual es el único consuelo. Este se basa en la empatía, en la compasión y permitir que se practiquen las creencias religiosas a través de la oración, el culto a imágenes sagradas y el sacramento del bautismo, pues así los familiares pueden enfrentar esta trágica situación y se evitan duelos patológicos.

Por otro lado, es importante indicar que todas las investigadoras de este estudio tienen experiencia en la investigación cualitativa, cuatro de ellas son docentes universitarias y asesoras de tesis, mínimamente han realizado un estudio en el área de neonatología y en general relacionado con la espiritualidad. Además, una de las autoras es enfermera neonatóloga y trabaja en la institución donde se realizó la investigación, pero no formó parte de la muestra, ni participó en la recolección de los datos.

Según lo anterior, esta investigación tuvo como objetivo describir la perspectiva de las enfermeras 
neonatólogas sobre el acompañamiento espiritual al familiar ante la muerte de un paciente neonato.

\section{MATERIAL Y MÉTODO}

Esta investigación cualitativa descriptiva se realizó en la UCIN de un hospital público de la ciudad de Chiclayo, Perú. La muestra por conveniencia se definió según el siguiente flujo: El servicio contaba con un total de 17 enfermeras y teniendo como criterio de inclusión el que estuvieran laborando como mínimo 3 meses en dicho servicio y como exclusión a aquellas que solo cubrían turnos, el grupo se redujo a 16; las enfermeras prefirieron que la entrevista se realizara al finalizar los turnos de tarde o de noche, pero tres de ellas no aceptaron participar puesto que no disponían del tiempo necesario, entonces, se comenzó con 13 profesionales y el grupo definitivo de participantes se completó con la saturación de los datos brindados por 12 enfermeras neonatólogas que laboraban en dicho nosocomio.

La recolección de los datos se realizó entre agosto y septiembre de 2019, a través de una entrevista semiestructurada ${ }^{(12)}$, guiada por la siguiente pregunta orientadora: ¿En qué consiste el acompańamiento espiritual que brindan a los padres/madres cuando fallece su hijo en la UCIN? Adicionalmente, se formularon otras preguntas para profundizar en el tema: ¿Qué actividades religiosas realizan o permite que los padres/madres realicen, ante la inminente muerte de su hijo, y porqué lo hacen? ¿Cómo es su trato con los padres/ madres cuando fallece su hijo? ¿Qué dificultades ha tenido para acompañar espiritualmente a los padres/madres cuando fallece su hijo?

El instrumento que contenía las preguntas fue elaborado por dos investigadoras y luego validado por juicio de expertos representados por 4 enfermeras especialistas en el tema y en investigación cualitativa. Luego de la aprobación del Comité de Ética en Investigación de la Facultad de Medicina de la Universidad Católica Santo Toribio de Mogrovejo (Resolución No 438-2019-USATFMED) y para evaluar la comprensión de las preguntas por las participantes del estudio, se realizó la prueba piloto en un hospital público de Lambayeque, con características similares al lugar de estudio. Teniendo la autorización del hospital público de Chiclayo para la ejecución del estudio, una investigadora procedió a la inmersión en el escenario, previa coordinación con la enfermera jefa del servicio, quien apoyó como contacto para que las enfermeras participaran. Luego de explicarles los objetivos de la investigación y según su disponibilidad, se acordó la fecha y la hora para realizar la entrevista. Cada entrevista duró aproximadamente 25 minutos, y se utilizó un grabador de voz previo consentimiento informado. Asimismo, después de transcribir las entrevistas se regresó al lugar de la investigación para confirmar los hallazgos y revisar datos particulares. Asimismo, los datos fueron procesados de manera manual para el análisis de contenido ${ }^{(13)}$, describir y/o interpretar los datos mediante tres fases: 1) Preanálisis: Se organizaron las entrevistas y se identificaron las similitudes y divergencias entre ellas, así mismo se fue remarcando las ideas relacionadas con los objetivos de investigación; 2) Codificación: Se fueron agrupando las ideas principales para organizar los discursos en una idea central o código de identificación. Existieron varios codificadores independientes que coincidieron obteniendo el mismo o similar resultado hasta formar las categorías. 3) Categorización: Se establecieron relaciones entre los códigos y se realizó la agrupación de la información por similitud, identificándose dos categorías ilustradas con los discursos más significativos. Para garantizar su confidencialidad se les asignó los códigos ENF1 hasta ENF12.

En el desarrollo de la investigación se respetaron los principios éticos de la Declaración de Helsinki de la Asociación Médica Mundial ${ }^{(14)}$. Las enfermeras no fueron expuestas a ningún daño físico, y para enfrentar cualquier riesgo mínimo de tipo emocional que la entrevista podía desencadenar al evocar recuerdos tristes de esta experiencia, tuvo en cuenta detener la entrevista, mostrar empatía, escucharla y darle un toque terapéutico, sin embargo, no hubo necesidad de ello, puesto que ninguna enfermera neonatóloga se mostró afectada. Asimismo, se tuvo en cuenta los criterios de rigor científico, según Rojas y Osorio ${ }^{(15)}$ : Credibilidad, permitió evidenciar el fenómeno de estudio y las experiencias humanas, tal y como son percibidos por los sujetos; Confirmabilidad, ya que la interpretación de la información garantizó la veracidad por los participantes del estudio y para ello fue necesario un registro de ideas o fuentes 
teóricas; por último, el criterio de Transferibilidad, que consistió en comparar los resultados de la investigación con otros contextos similares.

\section{RESULTADOS}

Las enfermeras tuvieron en promedio 40 años de edad y 10 años de experiencia en neonatología, solo una no profesaba la religión católica y dos no tenían hijos.

\section{a) Trato humano, empatía y prácticas religiosas previo al deceso}

En un primer momento las enfermeras observan que los padres presentan situaciones de angustia, miedo y mucho sufrimiento al enfrentar la muerte inminente de su bebé; esto constituye un suceso difícil de asimilar y necesitan que el personal de salud, en especial las enfermeras, brinden un trato humano pese a que cumplen múltiples funciones. En este estudio, las enfermeras acompañan al familiar del neonato crítico mediante la escucha activa y el toque terapéutico a través de una palmadita o "un abrazo" y esto significa, tácitamente, "te acompaño en tu dolor". Al respecto refieren:

"Las madres están sufriendo y tienen miedo porque sus bebés mueran...lloran, las apoyamos con un abrazo para tratar que se desahogue" ENF4. "Las madres lloran, les coloco una silla, les hablo, las escucho... están sintiendo por el miedo porque se acerca la muerte de su bebé... aunque sentimos que el tiempo es insuficiente por la cantidad de pacientes $y$ procedimientos" ENF7.

Al parecer, las enfermeras que son madres son más empáticas, sin duda tienen un trato más cálido porque sienten y se ponen en el lugar como madre. Lo manifiestan así:

"Tratamos de ponernos en el lugar de la mamá que está sufriendo, porque su bebé en cualquier momento puede morir..." ENF6. "Me da mucha tristeza y hasta ganas de llorar porque también soy madre y en su lugar me sentiría fatal... tratamos de decirle con una mirada cálida y un abrazo, estoy contigo, y rezamos para que encuentren paz" ENF10.

Un aspecto importante y que reconforta a las madres, es el rezar con ellas y sobre todo permitirle que estén junto a su niño hasta que ocurra el deceso:
"Rezamos juntas, tratamos de calmar a la mamá explicándole que esté tranquila, que si su niño ya está agónico pues hay que entregarlo al Señor y por algo suceden las cosas, que será un angelito" ENF2. "Cuando el bebé esta al final de la vida, dejamos a la madre que este de forma permanente con el bebé, dejándola que lo alce, que le hable, que le cante, que lo acaricie, que lo toque..." ENF3.

Además de rezar, y de permitir que la madre acompañe a su bebé, realizan el sacramento del bautismo. Esta es una práctica religiosa realizada por una autoridad eclesiástica de acuerdo a sus costumbres establecidas; de esta manera lo invocan al camino espiritual, haciendo partícipe a los padres y dando un nombre al niño:

"Si el niño está muy grave traen al sacerdote o al pastor para que lo bautice, claro que con todas las medidas de bioseguridad.... en el bautizo nosotras acompañamos...rezamos un padre nuestro, bautizan al niño o si está en agonia les dan los Santos Oleos" ENF6. "Dejamos pasar al sacerdote, a veces está prohibido, pero hacemos excepciones para que reciban el bautismo... a veces las madres nos piden que entre el pastor de su religión para que él sea quien ore por la salud del bebé o lo bautice" ENF12.

Las enfermeras también bautizan cuando no hay presencia de un sacerdote o un pastor, ya sea cuando el neonato se encuentre en un estado de agonía o cuando ya es evidente su partida. De esa manera apoyan espiritualmente, no solo a la madre, sino también a su hijo en los últimos segundos de vida:

"Cuando el sacerdote no puede venir, nosotras los bautizamos previa autorización de la madre y le pregunto el nombre que quiere ponerle...nosotros hacemos el bautizo, rezamos al niño, le colocamos el agua bendita en la frente, en el cuerpo y rezamos una oración junto con el personal que está en el momento" ENF2.

Por consiguiente, afirmamos que el acompañamiento espiritual no involucra un procedimiento físico, sino un sentido más amplio y profundo para enfrentar el sufrimiento y dolor por la muerte de un hijo. Es necesario valorar y respetar las creencias religiosas, transmitir esperanza accediendo al bautizo del neonato, la oración o la asistencia de una autoridad religiosa, así el cuidado espiritual 
se convierte en una prueba transcendente para la enfermera y el familiar en la UCIN.

\section{b) Compasión y consuelo durante el falleci- miento}

Las enfermeras del estudio reconocen que cuando el recién nacido fallece, afloran en los padres sentimientos de mucho sufrimiento y tristeza. En ese momento se necesita del acompańamiento de la enfermera, de mucha compasión, consuelo y resignación, es decir de apoyo espiritual. Se rescata que en estos momentos solo la fe reconforta y ayuda en el afrontamiento de la trágica pérdida, como es la muerte de un hijo. Tal experiencia se manifiesta a continuación:

"Es un momento muy triste cuando se le dice a la madre que falleció su bebé, no existen palabras que alivien o consuelen a las madres, por eso las escuchamos o abrazamos, dejamos que ellas expresen su dolor, por ejemplo, llorar, dejarlas que estén un momento con su bebé, y rezamos" ENF1. "Si está muy grave o falleció su bebé, debemos situarnos en el lugar de la madre y abrazarla, rezar y dejarla que se exprese y llore, expresando lo que siente" ENF5. "Cuando fallece el bebé solo un abrazo, o darles un vaso de agua, y permitirle que esté con su bebé antes de darle atención post mortem, ayudar con el trámite administrativo..." ENF7.

La muerte de un hijo es considerada una experiencia devastadora para los padres; asimismo, para el personal de salud y frente a esa situación, la enfermera busca mitigar el dolor de la madre y le brinda muestras afectivas como un abrazo, dar un vaso de agua, rezar por el descanso en paz del bebé, ayudar con los trámites, etc. Manifiestan que no existen palabras que reconforten esos trágicos momentos, pero justo en estos momentos es donde se hace más necesario el apoyo espiritual.

\section{DISCUSIÓN}

Las enfermeras son conscientes que perder un hijo no tiene nombre, no es algo común o esperado, los padres piensan que van a morir antes que sus hijos, pero qué sucede cuando la situación es al revés. Cuando ocurre la muerte de un hijo es un evento difícil de sobrellevar, los padres se sienten destrozados, en especial la madre y su único consuelo y refugio es aferrarse a Dios y a la creencia de que su pequeño estará en manos de él, todo unido a la empatía y trato humano del personal de la salud.

En este estudio los padres necesitaban de un trato humano ante la inminente muerte de su hijo, es común que lloren y encuentren palabras de alivio, pero la sola presencia, la escucha activa o un toque terapéutico significa "aquí estoy contigo y te comprendo". Tal vez son minutos o segundos en que el profesional de enfermería brinda un cuidado con empatía, trato amable, gestos y miradas compasivas, acciones sencillas que logran aliviar sentimientos y ser compañía hasta el último segundo de la vida de su hijo/a. Estos son resultados similares al estudio de Almeida, Moraes y Cunha ${ }^{(10)}$, quienes reconocían que la muerte del recién nacido es el momento más difícil para la familia. Por esta razón las enfermeras brindan intervenciones de escucha y tacto, respetando el silencio para ayudar a enfrentar la dolorosa situación, ofreciendo un ambiente cálido y enfatizando en la importancia de conseguir un lazo de confianza con el familiar. Ante esta situación es importante conservar un trato humano, informar, permitir que las madres sean escuchadas con una actitud amorosa y hospitalaria que genera mayor satisfacción con el cuidado ${ }^{(16)}$.

Cuando el profesional de enfermería se acerca a los familiares y muestra un trato humano de confianza y amor, estas expresiones permiten brindar un cuidado espiritual y conocer sus creencias y actividades en torno a la $\mathrm{fe}^{(17)}$. Aun así, hay estudios como el de Cortezzo, Sanders, Brownell y Moss ${ }^{(18)}$, que señalan que los padres de los neonatos fallecidos expresan falta de atención y cuidado por parte del profesional de enfermería, pues necesitaban más información, mejor disposición para atender el proceso de duelo y se sintieron incómodos en el cuidado de sus hijos al momento de morir. Se observa entonces que en el campo de enfermería el proceso de cuidar al final de la vida no es algo sencillo y no todos los profesionales se encuentran capacitados y preparados para atender dicho acontecimiento. Este cuidado va más allá de procedimientos y protocolos, involucra intervenciones espirituales que deben movilizar los recursos de sanación interna de los pacientes y familiares. Cuidar al final de la vida debe dejar de ser un tabú en nuestra profesión, se debe descubrir el sentido profundo 
que tiene esta etapa que es una más de la vida.

Por otro lado, las enfermeras que son madres son más empáticas y sensibles con los familiares al final de la vida de un niño y todas ellas permiten las prácticas religiosas como la oración y el bautismo. También se afectan psíquica y emocionalmente por este suceso, ocasionando en ellas un estado de sufrimiento, sobre todo cuando establecen una estrecha relación y afecto con los familiares. Dicho sufrimiento lo expresan llorando o rezando por el momento difícil que atraviesan los familiares y son algunas medidas utilizadas por los profesionales que enfrentan la situación ${ }^{(10)}$. Además, para las madres, dicho sufrimiento y dolor se incrementa cada día por la hospitalización que les impide sostener a su hijo en sus brazos. Son días llenos de angustia, intranquilidad, tristeza, incertidumbre por falta de información del neonato, por ello necesitan palabras de consuelo frente a la situación en la que viven ${ }^{(19)}$.

Coincidiendo con lo anterior, las enfermeras de este estudio manifestaron que brindan un acompañamiento espiritual, pues además de brindar un trato cálido, rezan con las madres y sobre todo les permiten que estén con sus niños hasta el final de la vida. Para Quinn ${ }^{(4)}$, el proceso de establecer y mantener relaciones compasivas con los padres ante la pérdida irreparable de su hijo, brindándoles un abrazo y palabras de consuelo, permite llevar el proceso de duelo de una manera positiva. Las enfermeras al conectarse con los padres, tanto emocional como espiritualmente, desempeñan un papel indispensable al iniciar y mantener una relación de cuidado compasivo y ayudan a sobrellevar la dolorosa muerte de su hijo.

Se logró evidenciar que cuando el recién nacido está en condiciones críticas o en un estado agonizante, las enfermeras sugieren a las madres que bauticen al neonato o, a veces, las mismas madres lo solicitan. Este sacramento lo celebra el sacerdote o un líder religioso, según el dogma de la familia. Sin embargo, hay enfermeras que realizan el ritual del bautizo cuando el niño está agonizando, porque no hay presencia del sacerdote o del pastor. Para ello, rocían agua bendita o agua natural por el cuerpo del bebé con la expresión de oraciones simbólicas. Esto se convierte en un acto reconfortante para las madres, porque a la vez, se les permite el contacto con su hijo y la práctica de sus creencias religiosas. Esto lo reafirma Guerrero ${ }^{(20)}$, quién establece que si la presencia de la muerte es inminente, la enfermera debe abogar por el neonato con entereza y evitar el encarnizamiento terapéutico, debe apoyar a los padres y trascender en el cuidado, administrando el bautizo para rescatarlo a la vida eterna. Además, Camacho-Ávila et al. ${ }^{(5)}$, nos mencionan que los rituales, en el momento o después de la muerte, son muy importantes para los familiares, así como el bautizar a los bebés, acto muy valorado que hace que el proceso de duelo sea más llevadero.

La realidad descrita es semejante a la de este estudio, pues la gran mayoría de las enfermeras son católicas y por ello se facilita el acceso de un sacerdote o pastor para realizar el sacramento del bautismo en compañía de sus padres. Además este acto religioso genera bienestar y paz a los padres, porque sus hijos son encomendados a Dios como "angelitos". Según Broadhurst y Harrington ${ }^{(17)}$, los efectos de los fenómenos trascendentales conducen a una sensación de bienestar y consuelo espiritual a los familiares. Asimismo, en la perspectiva cristiana, así como es frecuente encontrar sentimientos negativos, también se cultivan sentimientos de esperanza, reencuentro y de creer que el ser querido vivirá en el "cielo"(21). De este modo, el cuidado de un niño enfermo terminal solicita la participación a la vida divina en el Bautismo ${ }^{(22)}$.

La muerte es una cuestión muy difícil de aceptar, un momento crucial tanto para el paciente como para los familiares, por esta razón se hace muy necesario el acompañamiento o presencia amorosa del profesional de enfermería. Se rescata en el estudio que cuando el recién nacido fallece, la enfermera se muestra sensible ante el dolor de la madre, y le brinda muestras afectivas: un abrazo, un vaso de agua y rezos por el descanso en paz de su hijo y ayudar con los trámites administrativos, etc. Si esto no ocurre, tal como lo describen CamachoÁvila et al. ${ }^{(5)}$, es decir, cuando la enfermera no atiende sus necesidades o no se pone en el lugar de ellos para dar información, los padres perciben un dolor más profundo por la muerte del neonato. Ante ello, los padres sienten soledad, tristeza y su mundo se desborda. Asimismo, este proceso es más soportable para los padres cuando llevan a cabo rituales de acuerdo a su cultura y creencias porque decir adiós a su hijo es un momento de recogimiento.

Finalmente, la muerte del neonato conlleva a mucho sufrimiento y dolor; es un momento 
devastador en el que se hace muy necesaria la presencia de la enfermera involucrando prácticas religiosas y/o espirituales. Asimismo, debe brindar un trato humano, empático y compasivo a los familiares hasta el último segundo de vida de su hijo, es decir, cuidar con afecto, consolar a los padres, "estar en presencia", con una caricia, un abrazo o un apretón de manos. En efecto, el acompańamiento espiritual es un arte que se convierte en un cuidado transcendente para la enfermera y el familiar.

\section{CONCLUSIONES}

La muerte de un hijo en la Unidad de Cuidados Intensivos Neonatales es un suceso funesto $\mathrm{e}$ inexplicable para los padres y las madres lloran y no encuentran palabras de alivio.

A partir de los discursos de las enfermeras de UCIN se pudieron identificar dos categorías de acompańamiento espiritual: a) Trato humano, empatía y prácticas religiosas, previo al deceso y b) Compasión y consuelo durante el fallecimiento. Se muestran empáticas, compasivas y brindan consuelo a través de la esperanza en un ser superior. También dan un trato humano a través de la presencia, un abrazo, la escucha activa o un toque terapéutico y el permitir que la madre permanezca

\section{REFERENCIAS}

1. Organización Mundial de la Salud. Neonatal mortality rate [Internet]. 2018 [citado 1 may 2020]. Disponible en: https://www.who.int/gho/ child_health/mortality/neonatal/en/

2. Instituto Nacional de Estadística e Informática. Encuesta Demográfica y de Salud Familiar 2018 [Internet]. ENDES 2018. [citado 1 may 2020]. Disponible en: https://www.inei.gob.pe/media/ MenuRecursivo/publicaciones_digitales/Est/ Lib1656/index1. Html

3. Nascimiento T. Enfermería en la unidad de cuidados intensivos neonatal: asistencia del recién nacido de alto riesgo. $5^{\mathrm{a}}$ ed. Buenos Aires: Médica Panamericana; 2016. 356 p.

4. Quinn C. Creating and maintaining compassionate relationships with bereaved parents after perinatal unos momentos con su bebé hasta el final de la vida, antes de realizar la atención post mortem y los trámites administrativos. Esto reconforta y da fortaleza a los padres.

Se recomienda a los profesionales de la salud, que frente a un neonato que está al final de la vida, desempeñen una práctica ética, humanizada, con perspectiva biopsicosocial y cultural. De igual forma, considerar la espiritualidad como eje transversal en la formación académica del profesional de enfermería, tanto a nivel de pregrado y posgrado. Finalmente, es necesario continuar las investigaciones sobre el acompañamiento espiritual porque es la base del cuidado humano en enfermería. No se puede desligar al ser humano, entendido antropológicamente, del aspecto espiritual que es el que se rescata en estos momentos existenciales de sufrimiento. Para fortalecer este cuidado, es importante investigar hasta qué punto se consideran las creencias y prácticas en torno a la religión de los padres/madres que enfrentan la pérdida de un hijo.

Durante la recolección de datos se tuvo la limitante de la disponibilidad de tiempo por parte de las enfermeras, y por ello se tuvo que retornar varias veces al escenario de estudio. Este estudio no tuvo financiamiento y puede ser replicado en cualquier entidad de salud pública con UCIN. Las autoras declaran no tener conflictos de interés. death. Br J Midwifery [Internet]. 2016 Ago [citado 1 may 2020]; 24(8): 562-6. Disponible en: https:// doi.org/10.12968/bjom.2016.24.8.562

5. Camacho-Ávila M, Fernández-Sola C, JiménezLópez R, Granero-Molina J, Fernández-Medina I, Martínez-Artero L, et al. Experience of parents who have suffered a perinatal death in two Spanish hospitals: a qualitative study. BMC Pregnancy Childbirth [Internet]. 2019 [citado 1 may 2020]; 19(512): 1-11. Disponible en: https:// doi.org/10.1186/s12884-019-2666-z

6. Martínez C. El profesional de enfermería ante el duelo por muerte perinatal. Enferm Inv [Internet]. 2018 [citado 1 may 2020]; 3(1): 10-15. Disponible en: http://revistas.uta.edu.ec/erevista/index.php/ enfi/article/view/414/239.

7. Valdez Y, González J, Mascorro E. Espiritualidad en las Profesiones de Ayuda: Del Debate a la 
Integración. La Espiritualidad en los Procesos de Duelo. Puerto Rico: Ediciones Psicoespiritualidad; 2016. 210p.

8. Meert K, Thurston C, Briller S. The spiritual needs of parents at the time of their child's death in the pediatric intensive care unit and during bereavement: A qualitative study. Pediatr Crit Care Med [Internet]. 2005 Jul [citado 1 may de 2020]; 6(4): 420-7. Disponible en: https://doi. org/10.1097/01.PCC.0000163679.87749.CA

9. Noguera N, Pérez C. La situación de enfermería: "un milagro de vida" en la aplicación integral del concepto de duelo. Av en enfermería [Internet]. 2014 [citado 1 may 2020]; 32(2): 306-13. Disponible en: http://dx.doi.org/10.15446/ av.enferm.v32n2.46245

10. Almeida FA, Moraes MS, Cunha ML. Taking care of the newborn dying and their families: Nurses' experiences of neonatal intensive care. Rev Esc Enferm USP [Internet]. 2016 [citado 1 may 2020]; 50 Spec: 122-9. Disponible en: http://dx.doi. org/10.1590/S0080-623420160000300018

11. Fitch M, Bartlett R. Patient Perspectives about Spirituality and Spiritual Care. Asia Pac J Oncol Enfermera [Internet]. 2019 [citado 1 may 2020]; 6(2): 111-121. Disponible en: http://dx.doi.org/ 10.4103/apjon.apjon_62_18_

12. Berenguera A, Fernández de Sanmamed MJ, Pons M, Pujol E, Rodríguez D, Saura S. Escuchar, observar y comprender. Recuperando la narrativa en las Ciencias de la Salud. Aportaciones de la investigación cualitativa. Barcelona: Institut Universitari d'Investigació en Atenció Primària Jordi Gol; 2014. 219p.

13. Burns N, Gray J, Grove S. Investigación en enfermería: desarrollo de la práctica enfermera basada en la evidencia. 6a ed. Barcelona: Elsevier; 2016. $531 \mathrm{p}$.

14. Asociación Médica Mundial. Declaración de Helsinki de la AMM - Principios éticos para las investigaciones médicas en seres humanos [Internet]. 2017 Mar [citado 27 sept 2020]. Versión PDF 4 p. Disponible en: https://www.wma.net/ es/policies-post/declaracion-de-helsinki-de-laamm-principios-eticos-para-las-investigacionesmedicas-en-seres-humanos/

15. Rojas X, Osorio B. Criterios de Calidad y Rigor en la metodología Cualitativa. Gaceta de Pedagogía [Internet]. 2017 [citado 27 sept 2020]; 36: 6274. Disponible en: https://www.researchgate.net/ publication/337428163_Criterios_de_Calidad_y_ Rigor_en_la_Metodologia_Cualitativa

16. Delgado-Guay M. Spirituality and religiosity in supportive and palliative care. Curr Opin Support Palliat Care [Internet]. 2014 Sep [citado 1 may 2020]; 8(3): 308-13. Disponible en: http://dx.doi. org/10.1097/SPC.0000000000000079

17. Broadhurst K, Harrington A. A Thematic Literature Review: The Importance of Providing Spiritual Care for End-of-Life Patients Who Have Experienced Transcendence Phenomena. Am J Hosp Palliat Med [Internet]. 2016 [citado 1 may 2020]; 33(9): 881-93. Disponible en: https://doi. org/10.1177/1049909115595217

18. Cortezzo DM, Sanders MR, Brownell EA, Moss K. End-of-Life Care in the Neonatal Intensive Care Unit: Experiences of Staff and Parents. Am J Perinatol [Internet]. 2015 Dic [citado 1 may 2020]; 32(8): 713-24. Disponible en: https://doi. org/10.1055 / s-0034-1395475.

19. Acosta-Romo M, Cabrera-Bravo N, BasanteCastro Y, Jurado D. Sentimientos que experimentan los padres en el difícil camino de la hospitalización de sus hijos prematuros. Un aporte al cuidado humanizado. Univ y Salud [Internet]. 2017 [citado 1 may 2020]; 19(1): 17-25. Disponible en: https://dx.doi.org/10.22267/rus.171901.65

20. Guerrero S. Cuidado personalizado al recién nacido prematuro: aportes desde el personalismo ontológico moderno [Tesis para optar al grado académico de doctor en enfermería] [Internet]. Chiclayo: Universidad Católica Santo Toribio de Mogrovejo; 2018. 103p. Disponible en: http:// tesis.usat.edu.pe/handle/20.500.12423/1895

21. Bermejo J. Ética Humanismo y Sociedad / Duelo y espiritualidad. Rev chil endocrinol diabetes [Internet]. 2012 [citado 1 may 2020]; 5(4): 175-6. Disponible en: http://revistasoched. cl/4_2012/7_4-2012.pdf

22. Congregación para la Doctrina de la Fe. Carta Samaritanus Bonus sobre el cuidado de las personas en las fases críticas y terminales de la vida [Internet]. 2020 Jul [citado 1 may 2020]. Disponible en: https://bit.ly/36hxrYL 